\title{
LMI-based Multiobjective Optimization and Control of Flexible Aircraft Using VCCTEF
}

\author{
Sean Shan-Min Swei*, Guoming G. Zhu ${ }^{\dagger}$, Nhan Nguyen ${ }^{\ddagger}$
}

\begin{abstract}
This paper considers the control of coupled aeroelastic aircraft model with Variable Camber Continuous Trailing Edge Flap (VCCTEF) system. The relative motion between two adjacent flaps is constrained and this actuation constraint problem is converted into an output covariance constraint problem, and therefore can be formulated using linear matrix inequalities (LMIs). A set of LMI conditions is derived for the design of an observer-based dynamic output feedback controller for VCCTEF configured aeroelastic aircraft model. The proposed controller is then applied to the NASA Generic Transport Model (GTM) for simulation, and the results demonstrate the efficacy of the proposed approach.
\end{abstract}

\section{Introduction}

Elastic wing shaping actuation in-flight has been proven to be effective in reducing induced drag and enhancing lift performance. By actively controlling the wing wash-out twist and wing bending deflection, local angle of attack can be changed in such a manner that can result in lower fuel burn during cruise and enhanced lift performance during take-off and landing. As a low drag distributed actuation concept, the design of Variable Camber Continuous Trailing Edge Flap system is proposed, ${ }^{1}$ which consists of multiple aerodynamic control surfaces throughout the aeroelastic wing and provides active wing shaping control capability to gain aerodynamic efficiency. Two sets of control actuators are employed in order to actuate the VCCTEF. The light-weight shaped memory alloy (SMA) is adapted for controlling the shape of the first two chordwise sections of the three-section VCCTEF. The third section is controlled by high bandwidth electric drive motor (EDM) and provides the needed active wing shaping control in-flight.

This paper presents the mechanical modeling of the VCCTEF system using the conventional spring-massdashpot as dynamic components, and they are incorporated into the overall aeroelastic aircraft model. As illustrated in Swei and Nguyen, ${ }^{2}$ the actuation of VCCTEFs is constrained in both relative deflection and deflection rate. This is due to the elastomer material placed in between the flaps that confines the motion of two adjacent flaps. The placement of elastomer is to provide piece-wise continuous wing motion, so as to achieve active wing shape control. However, the presence of elastomer poses a great challenge in designing an effective and stabilizing feedback controller subjected to the constraints of bounded deflection and rate of VCCTEF dynamics. Previous works on bounded input and input rate, for instance ${ }^{5-7}$ and the references therein, offer a good framework for control designers to embark on the implementation aspect of the proposed approaches. A simple "software limiter" idea was proposed in Hess and Snell. ${ }^{6}$ There, by introducing the derivative and limited integration blocks a commanded input signal can be re-generated. In $\operatorname{Lin}^{7}$ a bounded input and input rate problem description was given which, in a sense, prescribes how the actuator should be moving. In Schewchun and Feron ${ }^{5}$ a bounded control approach; known as extended high performance bounded (eHPB) method, was proposed, in which a higher order state-space system representation was obtained by augmenting the states with the control, and in this setup the new control to the augmented system is in fact the input rate. The bounded control problem was then converted into bounded state and control problem. Inevitably, the control structure in this case becomes a dynamic controller. The concept of pseudo-control hedging or $\mathrm{PCH}^{4,8}$ was another viable alternative control approach which was well suited for dealing with such practical control issues as actuator saturation. In Swei and Nguyen ${ }^{2}$ a PCH-based bounded

\footnotetext{
*Research Scientist, Intelligent Systems Division, NASA Ames Research Center

${ }^{\dagger}$ Professor, Mechanical Engineering, Electrical and Computer Engineering, Michigan State University

${ }^{\ddagger}$ Research Scientist, Intelligent Systems Division, NASA Ames Research Center
} 
input and input rate control approach was proposed by utilizing eHPB method, where an additional control loop was added through PCH model to re-shape the control command.

In this paper, we propose to develop a stabilizing controller for VCCTEFs equipped aeroelastic aircraft using output feedback. In particular, we seek to design a standard observer-based dynamic feedback controller. In this approach, differs from previous approaches which were mostly full state-feedback based, we first formulate the combined VCCTEFs and aeroelastic aircraft dynamics as state-space representation of a dynamical system with constrained outputs. In this setup, the constrained relative deflection and deflection rate of VCCTEF actuation is converted into constrained output components in the augmented system. The control design objective then becomes finding an observer-based output feedback controller that is stabilizing and solves the output constrained optimization problem. Since the maximum relative deflection and deflection rate between adjacent flaps is known apriori, the output constrained optimization problem can be cast as an optimization problem subject to $L_{2}$ to $L_{\infty}$ gain, which can then be solved as a generalized $\mathcal{H}_{2}$ performance problem, ${ }^{13}$ hence a standard solution; if it exists, can be applied. Furthermore, the existence of such solution can be determined by checking if a feasible solution exist to a coupled linear matrix inequalities. ${ }^{13}$ Therefore, the numerically efficient LMI-based convex optimization tools and algorithms can be applied for control analysis and synthesis.

As discussed, a dynamical system with bounded input and bounded input rate VCCTEF actuation can be naturally converted into the constrained output problem for augmented system of higher order. In addition to the output constraints that were emerged from VCCTEFs, in the case of flexible wing control, we also want to consider constraining the vibrational motion of the aeroelastic wing. Furthermore, for practical consideration, we want the feedback controlled system to possess a good robustness to unmodeled dynamics, disturbance rejection, etc. It is shown that this robustness problem can be cast into minimizing the $L_{2}$ to $L_{2}$ gain or $\mathcal{H}_{\infty}$ performance problem, and again the solution and its existence can be formulated in LMI. ${ }^{16-18}$ Therefore, the framework of the proposed aeroelastic aircraft control using the constrained VCCTEF actuation is a multiobjective optimal control design problem.

This paper is organized as follows. In Section II we describe the detail modeling of VCCTEFs and introduce the prescribed relative constraints on deflection and deflection rate between the flaps. Furtermore, the differences in deflection and deflection rate between neighboring flaps are treated as control outputs. Section III presents the aeroelastic aircraft dynamics and incorporates it with the VCCTEF dynamics. Some preliminary results on LMI characterization of output covariance control problem are given in Section IV, which provide a basis for designing an observer-based output feedback controller. In Section V the VCCTEF actuation constraints are converted into the output constraints in the combined system representation. The problem of multiobjective optimal control design is then formulated subject to $L_{2}$ to $L_{\infty}$ performance constraints as well as VCCTEF actuation constraints. Simulation results for the application of proposed controller to GTM aircraft at flutter condition is considered in Section VI. Concluding remarks are given in Section VII.

Throughout this paper, we make use of the following standard definition of $\mathcal{L}_{2}$ and $\mathcal{L}_{\infty}$ norms on $x(\sigma) \in$ $R^{k}$ for all $\sigma \geq 0,{ }^{10}$

$$
\begin{aligned}
& \|x\|_{2}^{2}:=\int_{0}^{\infty} x^{t}(\sigma) W^{-1} x(\sigma) d \sigma \\
& \|x\|_{\infty}^{2}:=\sup _{\sigma \geq 0} x(\sigma)^{t} x(\sigma)
\end{aligned}
$$

where $W>0$ is a weighting matrix. Accordingly, we can define the $\mathcal{L}_{2}$ disturbance set as follows,

$$
\mathcal{S}:=\left\{x: R \rightarrow R^{k} \text { and }\|x\|_{2}^{2} \leq 1\right\} .
$$

\section{Modeling of VCCTEFs}

Figure 1 shows a GTM aircraft configured with VCCTEFs. Figure 2 illustrates the detail constructs of a VCCTEF that shows each flap has three segment sections. Sections 1 and 2 are driven by shape memory alloy (SMA) and produce high lift, whereas section 3 is driven by high bandwidth electric drive motor (EDM) necessary to provide active wing shaping control. At nominal cruise flight, we consider only the section 3 is being activated.

In order to achieve active wing shaping control for drag reduction in flight, it should be noted that each VCCTEF can not be operated independently. Therefore, to ensure and approximate smooth and continuous variation of wing shape, it is necessary to confine the relative motion between the two neighboring flaps. To achieve this, an elastomer material is placed between two adjacent flaps, which produces continuous 
transition for relative motion. However, the presence of elastomer also introduces additional constraints on the performance of VCCTEF, and these constraints are needed to be addressed and incorporated in the control systems design process. The maximum relative flap motion is often prescribed apriori and can be used for control analysis and synthesis. Figure 3 shows the schematics of the 3rd segment of the two adjacent VCCTEFs with the elastomer being modeled as dashpot-spring mechanism, where $(C, K)$ denotes the elastomer damping and stiffness properties. As shown, the relative deflection between, for example, $\theta_{1}$ and $\theta_{2}$ is constrained, as well as the relative rate between $\dot{\theta}_{1}$ and $\dot{\theta}_{2}$. In addition, the individual flap deflection $\theta_{i}$ and deflection rate $\dot{\theta}_{i}$ are also constrained. It is important to note that the elastomer material properties would depend on the ambient conditions. In this study, we shall use notional material properties; however, they can be considered as potential design parameters for future research.

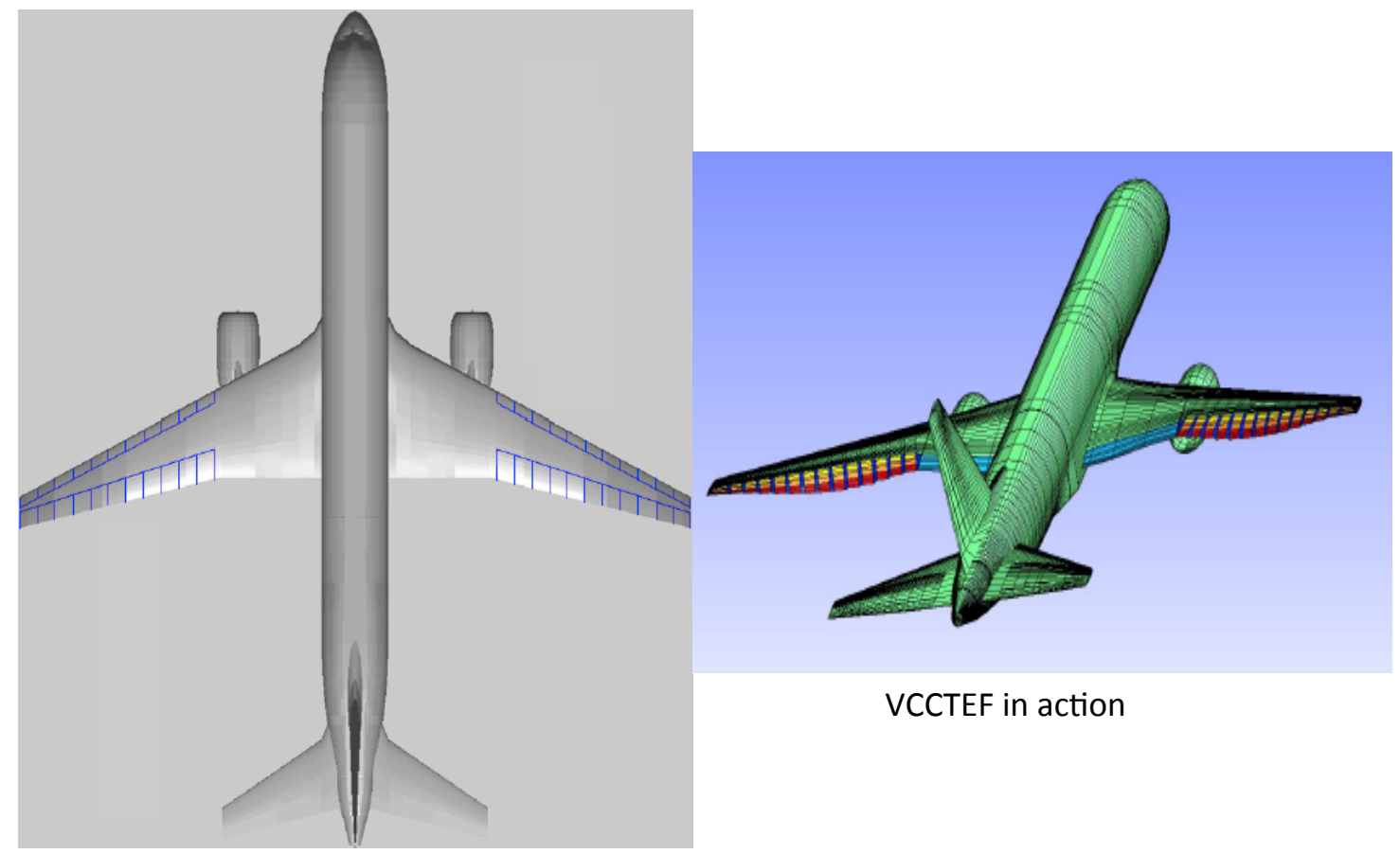

Figure 1. GTM with equally spaced VCCTEFs

Since the EDM actuator has much higher bandwidth, the frequency response analysis technique can be applied to approximate the dynamic model at the frequency range of our interest, and we assume that this model is a second order of the form

$$
M \ddot{\theta}_{i}+C \dot{\theta}_{i}+K \theta_{i}=\delta_{i} ; i=1,2, \cdots, m,
$$

where $\theta_{i}$ and $\dot{\theta}_{i}$ are output of $i$ th actuator and represent respectively the deflection angle and deflection rate of $i$ th flap, and $\delta_{i}$ denotes the control command to the $i$ th flap. The parameters $(M, C, K)$ are known mass, damping and stiffness properties derived from the frequency domain system identification for an EDM actuator. We have assumed that all EDM actuators share the same dynamic characteristics, though it is not reflective of actual implementation, this assumption simplifies our subsequent problem formulation. We can further model the coupled dynamic behavior between the elastomer and the EDM deriven flaps, as shown in Fig. 4. It should be noted from Fig. 4 that, in general, $\delta_{i}$ and $\theta_{i}$ are not the same in actual implementation, and this is the case in particular because of the presence of elastomer. When all the $m$ flaps 


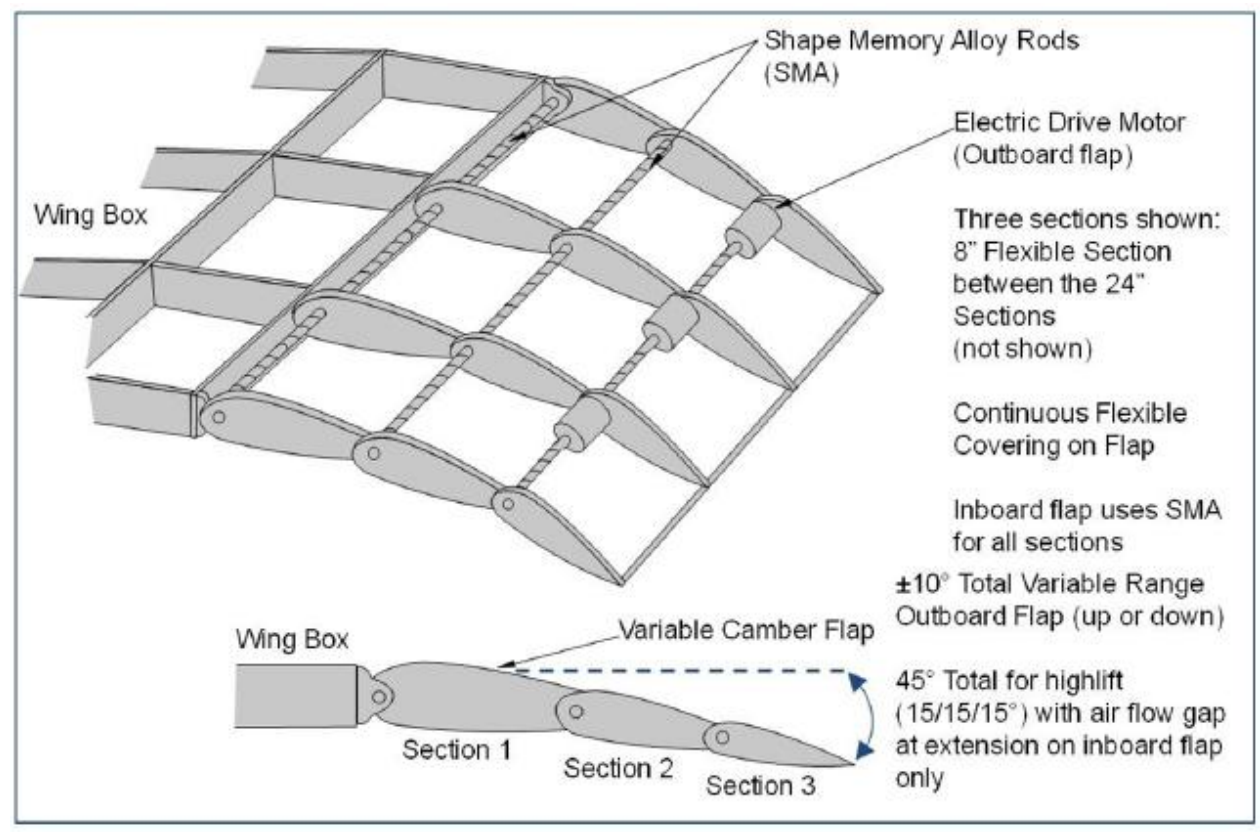

Figure 2. VCCTEF actuation using SMA and EDM

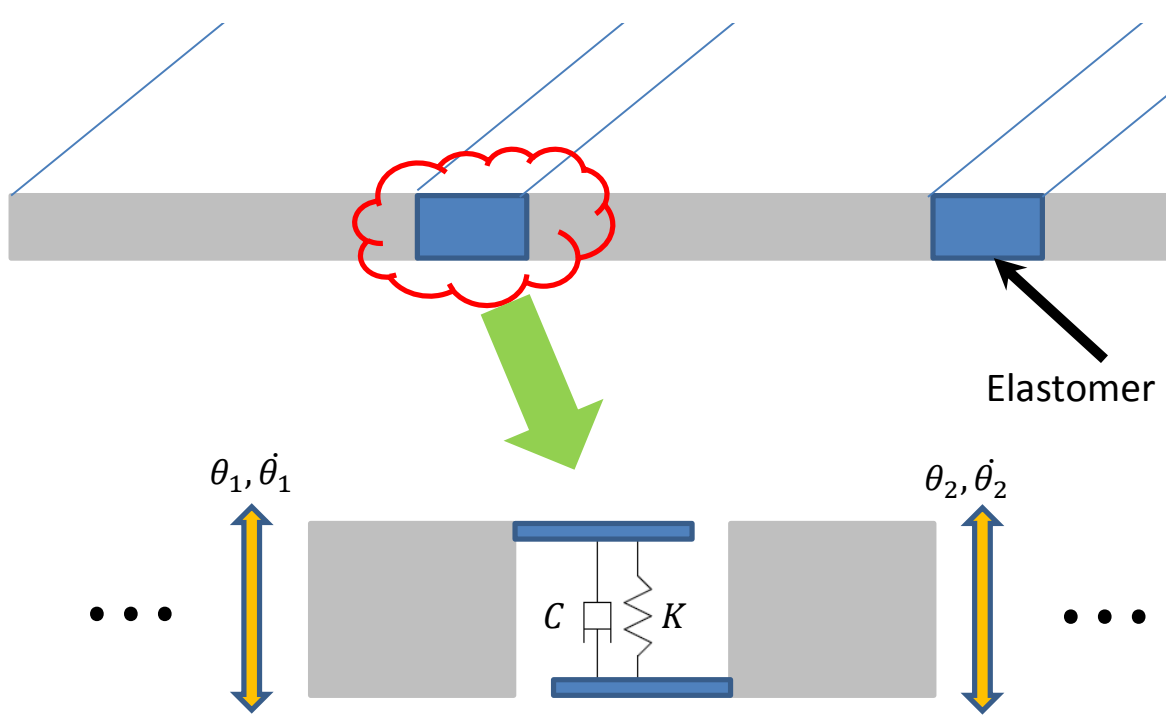

Figure 3. Schematics of VCCTEF 
are interconnected with $m-1$ elastomer, the overall combined actuator dynamics can be described by

$$
\begin{aligned}
& \underbrace{\left[\begin{array}{cccccc}
M & 0 & 0 & 0 & \cdots & 0 \\
0 & M & 0 & 0 & \cdots & 0 \\
0 & 0 & M & 0 & \cdots & 0 \\
\vdots & & \cdots & & \vdots \\
0 & 0 & 0 & 0 & \cdots & M
\end{array}\right]}_{M_{f}} \underbrace{\left[\begin{array}{c}
\ddot{\theta}_{1} \\
\ddot{\theta}_{2} \\
\ddot{\theta}_{3} \\
\vdots \\
\ddot{\theta}_{m}
\end{array}\right]}_{\ddot{\delta}_{f}}+\underbrace{\left[\begin{array}{ccccccc}
C+c & -c & 0 & 0 & \cdots & 0 \\
-c & C+2 c & -c & 0 & \cdots & 0 & 0 \\
0 & -c & C+2 c & -c & \cdots & 0 & \vdots \\
\vdots & & \cdots & & \vdots \\
0 & 0 & 0 & 0 & \cdots & C+c
\end{array}\right]}_{C_{f}} \underbrace{\left[\begin{array}{c}
\dot{\theta}_{1} \\
\dot{\theta}_{2} \\
\dot{\theta}_{3} \\
\vdots \\
\dot{\theta}_{m}
\end{array}\right]}_{\dot{\delta}_{f}}+ \\
& \underbrace{\left[\begin{array}{cccccc}
K+k & -k & 0 & 0 & \cdots & 0 \\
-k & K+2 k & -k & 0 & \cdots & 0 \\
0 & -k & K+2 k & -k & \cdots & 0 \\
\vdots & & \cdots & & & \vdots \\
0 & 0 & 0 & 0 & \cdots & K+k
\end{array}\right]}_{K_{f}} \underbrace{\left[\begin{array}{c}
\theta_{1} \\
\theta_{2} \\
\dot{\theta}_{3} \\
\vdots \\
\theta_{m}
\end{array}\right]}_{\delta_{f}}=\underbrace{\left[\begin{array}{c}
\delta_{1} \\
\delta_{2} \\
\delta_{3} \\
\vdots \\
\delta_{m}
\end{array}\right]}_{u}
\end{aligned}
$$

where $\delta_{f}$ denotes the flap deflection and $u$ the control input to VCCTEFs. In attaining the above we have assumed that all $m-1$ elastomers have the same material properties. As noted earlier, the flap deflection and its rate of deflection is bounded by $\phi_{i}$ and $d \phi_{i}(i=1, \cdot, m)$, respectively, and the relative deflection between two adjacent flaps is also constrained by $\alpha_{j}$, i.e. $\left|\theta_{j}-\theta_{j+1}\right| \leq \alpha_{j}(j=1, \cdot m-1)$. In practical application, the motion of VCCTEF is also confined by the relative angular deflection rate, i.e. $\left|\dot{\theta}_{j}-\dot{\theta}_{j+1}\right| \leq d \alpha_{j}$, where both $\alpha_{j}$ and $d \alpha_{j}$ are known constants. Now, we can represent (3) in a state-space form, and incorporate the constraints as controlled output, as follows,

$$
\Sigma_{f}:\left\{\begin{array}{l}
\dot{x}_{f}=A_{f} x_{f}+B_{f} u \\
y_{f}=H_{f} x_{f}
\end{array}\right.
$$

where $x_{f}=\left[\begin{array}{ll}\delta_{f}^{t} & \dot{\delta}_{f}^{t}\end{array}\right]^{t}$ denotes the state, $y_{f}$ the controlled output, and the system matrices $\left(H_{f}, A_{f}, B_{f}\right)$ are given by

$$
A_{f}=\left[\begin{array}{cc}
0 & I_{m} \\
-M_{f}^{-1} K_{f} & -M_{f}^{-1} C_{f}
\end{array}\right], B_{f}=\left[\begin{array}{c}
0 \\
M_{f}^{-1}
\end{array}\right], H_{f}=\left[\begin{array}{cc}
I_{m} & 0 \\
0 & I_{m} \\
H & 0 \\
0 & H
\end{array}\right],
$$

where $I_{m}$ denotes $m \times m$ identity matrix and $H \in R^{(m-1) \times m}$ is given by

$$
H=\left[\begin{array}{ccccccc}
1 & -1 & 0 & 0 & \cdots & 0 & 0 \\
0 & 1 & -1 & 0 & \cdots & 0 & 0 \\
\vdots & & \cdots & & & \vdots & \\
0 & 0 & 0 & 0 & \cdots & 1 & -1
\end{array}\right]
$$

It should be noted that the entries in $y_{f}$ are bounded by individual deflection and deflection rate, as well as the relative deflection and deflection rate between two adjacent flaps. In this paper, we consider that all VCCTEFs have the same deflection and deflection rate constraints, and any two adjacent VCCTEFs have the same relative deflection and deflection rate constraints. Therefore, the VCCTEF actuation constraints are given as follows:

$$
\left\{\begin{array}{l}
\left|\left(y_{f}\right)_{i}\right| \leq \phi, i=1, \cdots, m \\
\left|\left(y_{f}\right)_{j}\right| \leq d \phi, j=m+1, \cdots, 2 m \\
\left|\left(y_{f}\right)_{k}\right| \leq \alpha, k=2 m+1, \cdots, 3 m-1 \\
\left|\left(y_{f}\right)_{l}\right| \leq d \alpha, l=3 m, \cdots, 4 m-1
\end{array}\right.
$$




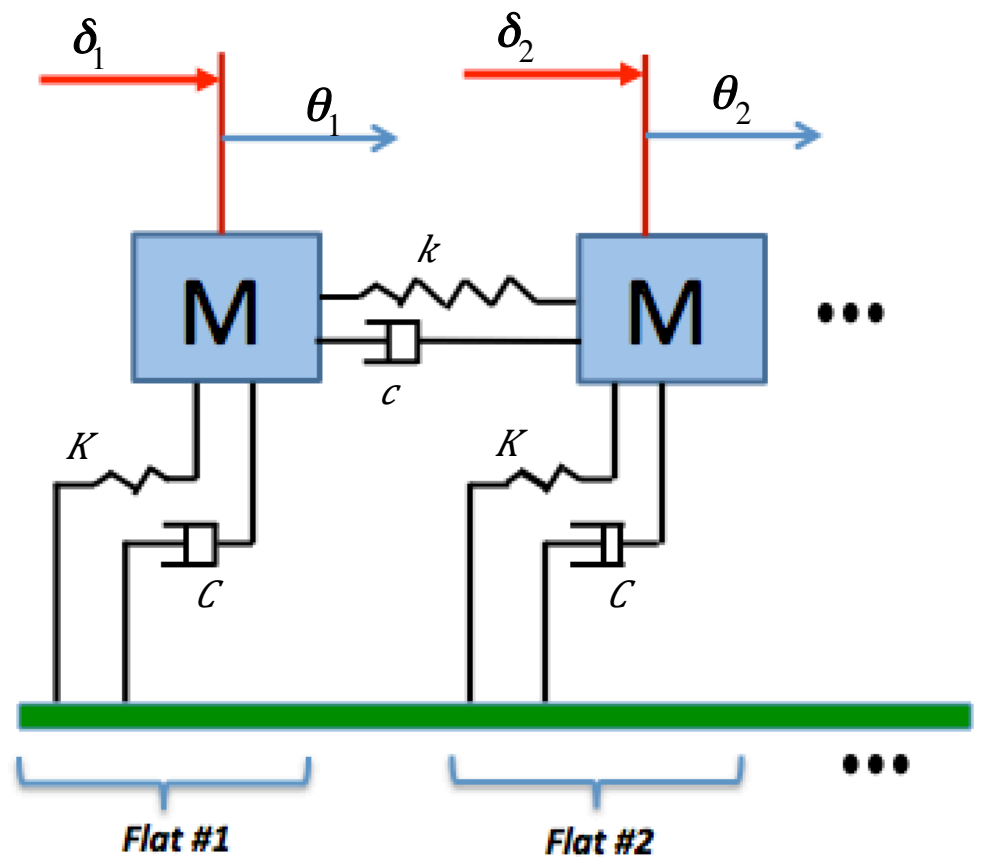

Figure 4. Interconnected dynamical model between EDM driven flaps and elastomer

\section{Aeroelastic Aircraft Dynamics with VCCTEFs}

Figure 1 shows the GTM with configured VCCTEF system. As shown, there are 11 flaps per wing and they are equally spaced. In this paper, we consider aircraft flight dynamics and aeroelastic wings at cruise conditions. The equations of motion for coupled aircraft rigid-body dynamics with flexible aeroelastic wing modes at nominal cruise flight can be described by

$$
\underbrace{\left[\begin{array}{c}
\dot{x}_{a}(t) \\
\dot{x}_{e}(t)
\end{array}\right]}_{\dot{x}_{s}(t)}=\underbrace{\left[\begin{array}{cc}
A_{a a} & A_{a e} \\
A_{e a} & A_{e e}
\end{array}\right]}_{A_{s}} \underbrace{\left[\begin{array}{c}
x_{a}(t) \\
x_{e}(t)
\end{array}\right]}_{x_{s}(t)}+\underbrace{\left[\begin{array}{c}
B_{a} \\
B_{e}
\end{array}\right]}_{B_{s}} \delta_{f}(t)+\underbrace{\left[\begin{array}{c}
D_{a} \\
D_{e}
\end{array}\right]}_{D_{s}} w(t)
$$

where $x_{a}$ denotes the aircraft's rigid-body states, $x_{e}$ consists of displacement and velocity of aeroelastic wing at generalized coordinates, ${ }^{3} \delta_{f}$ denotes the VCCTEF 3rd segment deflection, and $w \in R^{k}$ denotes the external disturbance, such as wind gust and $w \in \mathcal{S}$ as defined in (1). The matrices $A_{a a}$ and $A_{e e}$ of compatible dimensions contain aircraft rigid-body and aeroelastic characteristics, whereas $A_{a e}$ and $A_{e a}$ correspond to aeroelastic coupling and aircraft rigid-body coupling, respectively. Note that the dimension of overall system depends on the number of rigid-body states and number of aeroelastic modes included in the problem setup. Throughout this paper, we will work with a reduced-order aeroelastic model. For detail derivation of (6) and the process for model reduction, readers may want to consult Nguyen et al. ${ }^{1,9}$ and Swei et al. ${ }^{3}$

Now, combining the aeroelastic aircraft dynamics (6) with VCCTEF actuator dynamics $\Sigma_{f}$ described in (4), and introducing the new controlled and measured outputs, $y_{p}$ and $z_{p}$, respectively, yields the following open-loop system description,

$$
\Sigma_{o}:\left\{\begin{aligned}
\dot{x}_{p}(t) & =A_{p} x_{p}(t)+B_{p} u(t)+D_{p} w_{p}(t) \\
y_{p}(t) & =C_{p} x_{p}(t) \\
z_{p}(t) & =M_{p} x_{p}(t)+N_{p} w_{p}(t)
\end{aligned}\right.
$$

where $x_{p}(t)$ denotes the combined states of $x_{s}(t)$ and $x_{f}(t)$, and $y_{p}(t)$ the controlled output variables of the form

$$
y_{p}(t)=\left[\begin{array}{l}
y_{s}(t) \\
y_{f}(t)
\end{array}\right]
$$


and $y_{s}(t)=C_{s} x_{s}(t)$ denotes the controlled outputs for aeroelastic aircraft, which for instance, might include wing deflection and deflection rate at various locations along the wing span that we want to monitor and suppress. In this regard, we have chosen $n_{s}$ number of controlled outputs for performance evaluation, hence $y_{s}(t) \in R^{n_{s}}$. The measurement outputs are denoted by $z_{p}(t)$ and, without loss of generality, we assume that the pair $\left(M_{p}, A_{p}\right)$ is observable. The combined disturbance vector $w_{p}(t)$ consists of $w(t)$ and $v(t)$, where $v(t)$ denotes the measurement white noise with intensity $V>0$. The system matrices in (7) are given by

$$
\begin{aligned}
& A_{p}=\left[\begin{array}{cc}
A_{s} & B_{s} E \\
0 & A_{f}
\end{array}\right], B_{p}=\left[\begin{array}{c}
0 \\
B_{f}
\end{array}\right], C_{p}=\left[\begin{array}{cc}
C_{s} & 0 \\
0 & H_{f}
\end{array}\right], \\
& D_{p}=\left[\begin{array}{cc}
D_{s} & 0 \\
0 & 0
\end{array}\right], N_{p}=\left[\begin{array}{ll}
0 & I
\end{array}\right], E=\left[\begin{array}{ll}
I_{m} & 0
\end{array}\right] .
\end{aligned}
$$

The complete system description that combines both the aircraft aeroelastic model and the VCCTEF actuation dynamics is described in (7), which is in the standard form of many control problems considered in the context of $H_{2}$ and $H_{\infty}$ optimization. It should be noted that, in attaining $\Sigma_{o}$, we have in fact converted the actuation constrained problem into output constrained problem for a system of higher dimension. Specifically, the controlled output $y_{f}$ in the combined system contains the VCCTEF constraints for each flap and pair of adjacent flaps, which are part of the states in the new system description. In addition, $y_{p}$ may represent bending and torsional deflections and deflection rates at particular locations along the aeroelastic wing that we want to monitor and suppress. Therefore, the control objective considered in this paper renders itself naturally as an Output Covariance Constraint (OCC) control problem. ${ }^{3,10}$ The problem of flutter suppression for aeroelastic wing with the conventional control surfaces was first considered in Swei et al. ${ }^{3}$ under the framework of OCC problem setup via a dynamic output feedback. In this paper, we consider OCC control problem for VCCTEF configured aeroelastic aircraft by utilizing the standard multiobjective optimization results that were characterized by LMIs. ${ }^{15}$ In particular, given the VCCTEF actuation constraints, we focus on solving the minimum achievable output covariance problem for aeroelastic aircraft. The next section provides some helpful results.

\section{Some Preliminary Results}

We first present the LMI characterization of the output covariance constraint problem. ${ }^{19}$ For this study, we consider the following system,

$$
\left\{\begin{array}{l}
\dot{x}=A x+D w \\
y_{i}=C_{i} x, i=1,2, \cdots, q
\end{array}\right.
$$

where $\left(C_{i}, A, D\right)$ are matrices of compatible dimensions and $w \in \mathcal{S}$. It is well known ${ }^{14}$ that, if $A$ is Hurwitz, the output covariance for (9) is given by

$$
Y_{i}=C_{i} X C_{i}^{t}, i=1,2, \cdots, q,
$$

where $X$ is the controllability Gramian matrix satisfying the following Lyapunov equation

$$
X A^{t}+A X+D W D^{t}=0
$$

Therefore, given the desired output covariance $\bar{Y}_{i}$, the output covariance constraint problem for (9) is solved when

$$
Y_{i} \leq \bar{Y}_{i}, i=1,2, \cdots, q
$$

Our goal is to characterize the output covariance constraint problem using LMI. For this, we let $\epsilon>0$ be given. Utilizing the monotonicity of Lyapunov solution, we have the following Lyapunov equation

$$
\bar{X} A^{t}+A \bar{X}+D W D^{t}+\epsilon I=0,
$$

where $\bar{X}$ is a unique positive definite solution. It is straightforward to show that $\bar{X}>X$. Therefore, (10) and (12) can be modified as

$$
C_{i} \bar{X} C_{i}^{t}<\bar{Y}_{i}, i=1,2, \cdots, q
$$


and (13) can be rewritten as

$$
\bar{X} A^{t}+A \bar{X}+D W D^{t}<0 .
$$

After applying the Schur complement argument to (15), we obtain the following LMI expression,

$$
\left[\begin{array}{cc}
\bar{X} A^{t}+A \bar{X} & D Q \\
Q D^{t} & -I
\end{array}\right]<0
$$

where $Q=W^{\frac{1}{2}}$. Note that (16) is an affine function in both $\bar{X}$ and $Q$, and defines a convex set of pairs $(\bar{X}, Q)$, so it can be solved efficiently using, for instance, interior-point methods from convex optimization. ${ }^{12}$ Therefore, the output covariance constraint problem can be summarized in the next lemma, ${ }^{19}$ whose proof follows readily from the arguments given above.

Lemma 1 Consider (9) and let $\bar{Y}_{i}>0, i=1,2, \cdots, q$, be given. Then, the output covariance constraint problem is solvable, if there exists $\bar{X}$ satisfying

(i) $\bar{X}>0$;

(ii) (16) is feasible;

(iii) $C_{i} \bar{X} C_{i}^{t}<\bar{Y}_{i}, i=1,2, \cdots, q$.

Conditions (i) and (ii) imply that $A$ is Hurwitz. Note that (iii) provides an upper bound for output covariance, since $\bar{X}>X$. As a corollary, the "maximum allowable" disturbance $W$ (or $Q$ ) that ensures (i) - (iii) can also be attained from Lemma 1 with a slight modification.

Corollary 1 The output covariance constraint problem with maximum allowable disturbance is solvable, if there exists $\bar{X}$ that maximizes

$$
\max _{\bar{X}, Q} \rho^{2}
$$

subject to (i) - (iii) and

$$
\left[\begin{array}{cc}
-\rho^{2} I & Q \\
Q^{t} & -I
\end{array}\right] \leq 0 .
$$

Therefore, the purpose of Lemma 1 is two folds. First is that, for a known disturbance set $\mathcal{S}$, it provides a feasibility characterization of output covariance constraint problem for all $w \in \mathcal{S}$, and the second is that, in addition, it provides a measure of disturbance input. To illustrate this part, we consider (9) and establish the disturbance bound in terms of system properties and desired output covariance. For this study, since $A$ is Hurwitz, there exist constants $\eta_{i}>0$ and $\lambda>0$, such that

$$
\left\|C_{i} e^{A \sigma} D\right\| \leq \eta_{i} e^{-\lambda \sigma}, \text { for all } \sigma \geq 0,
$$

where $i=1,2, \cdots, q$. The matrix norm used above is the Euclidean norm. Furthermore, we let $W=\beta I$, where $\beta>0$ denotes the intensity of random disturbance $w$ and is to be determined. We claim that if the disturbance intensity $\beta$ satisfies

$$
\beta \leq \frac{2 \lambda}{\eta_{i}^{2}}\left\|\bar{Y}_{i}\right\|, i=1,2, \cdots, q,
$$

then $\left\|Y_{i}\right\| \leq\left\|\bar{Y}_{i}\right\|$. To show this, recall the Lyapunov equation (11). Since $A$ is Hurwitz, it can be $\operatorname{shown}^{11}$ that the unique solution to (11) is given by

$$
X=\int_{0}^{\infty} e^{A \sigma} D W D^{t} e^{A^{t} \sigma} d \sigma
$$

Now, substituting the above into (10) yields

$$
Y_{i}=\int_{0}^{\infty} C_{i} e^{A \sigma} D W D^{t} e^{A^{t} \sigma} C_{i}^{t} d \sigma
$$


for $i=1,2, \cdots, q$. Taking the norm on both sides renders

$$
\left\|Y_{i}\right\| \leq \int_{0}^{\infty}\left\|C_{i} e^{A \sigma} D\right\|^{2}\|W\| d \sigma .
$$

It now follows from (17) that

$$
\begin{aligned}
\left\|Y_{i}\right\| & \leq \beta \int_{0}^{\infty} \eta_{i}^{2} e^{-2 \lambda \sigma} d \sigma \\
& =\beta \frac{\eta_{i}^{2}}{2 \lambda} .
\end{aligned}
$$

Therefore, $\left\|Y_{i}\right\| \leq\left\|\bar{Y}_{i}\right\|$ if $\beta$ satisfies (18).

\section{LMI Characterization of Output Covariance Control Problem}

Recall (7). We have shown that by integrating VCCTEF dynamics with aeroelastic aircraft equations of motion, the bounded input deflection and deflection rate problem can be converted into OCC problem. In this section, we present the solution to output covariance control problem by utilizing LMIs and proposes a stabilizing controller if a feasible solution exists.

For systems of large dimensions, the control objectives and constraints are often prescribed with respect to a collection of states. Therefore, we can partition the controlled output $y_{p}$ into a series of block outputs $\mathrm{as}^{3}$

$$
y_{p}=\left[\begin{array}{c}
y_{1} \\
y_{2} \\
\vdots \\
y_{q}
\end{array}\right] ; y_{i}=\left(C_{p}\right)_{i} x_{p},
$$

where, in the case of aeroelastic wing control subject to actuation constraints, we may choose $y_{s}=\left[y_{1}^{t}, \cdots, y_{n_{s}}^{t}\right]^{t}$ to denote aeroleastic wing deflection and deflection rate along the wing span and $y_{f}=\left[y_{n_{s}+1}^{t}, \cdots, y_{q}^{t}\right]^{t}$ to represent the constraints on VCCTEF actuation. Now, we recall the combined aeroelastic aircraft equation with VCCTEF dynamics as given in (7), and substitute $y_{p}$ by the partition given in (20), we obtain

$$
\Sigma_{o}:\left\{\begin{array}{l}
\dot{x}_{p}(t)=A_{p} x_{p}(t)+B_{p} u(t)+D_{p} w_{p}(t) \\
y_{i}(t)=\left(C_{p}\right)_{i} x_{p}(t), i=1,2, \cdots, q \\
z_{p}(t)=M_{p} x_{p}(t)+N_{p} w_{p}(t)
\end{array}\right.
$$

In this paper, we focus mainly on developing an observer-based dynamic output feedback controller of the form,

$$
\left\{\begin{array}{l}
\dot{x}_{c}(t)=A_{p} x_{c}(t)+B_{p} u(t)+L_{c}\left(z_{p}(t)-M_{p} x_{c}(t)\right) \\
u(t)=K_{c} x_{c}(t)
\end{array}\right.
$$

where $L_{c}$ and $K_{c}$ are respectively the observer gain and full state feedback gain, and they are to be determined. The objective of this paper can then be stated as follows:

Given the VCCTEF actuation constraints, find the dynamic output feedback controller (22) such that it minimizes the vibrational motion of aeroelastic wings.

Theorem 1 Consider the system $\Sigma_{o}$ described in (21) and given the VCCTEF constraints as defined in (5). The minimum achievable output covariance control problem is solvable with the dynamic output controller given in (22), if there exist a matrix $G$ and a pair of positive definite symmetric matrices $Y$ and $Z$ that minimize the performance cost

$$
\min _{G, X, Z} \operatorname{trace}\left[\left(C_{p}\right)_{i}(Y+Z)\left(C_{p}\right)_{i}^{t}\right], i=1, \cdots, n_{s},
$$

subject to 
(i)

$$
\left[\begin{array}{cc}
A Y+Y A^{t}+D_{p} W D_{p}^{t} & Y M_{p}^{t} \\
M_{p} Y & V
\end{array}\right]<0,
$$

and define the observer gain $L_{c}$ as

$$
L_{c}=Y M_{p}^{t} V^{-1}
$$

(ii)

$$
\left[\begin{array}{cc}
A Z+Z A^{t}+G^{t} B_{p}^{t}+B_{p} G & L_{c} V^{\frac{1}{2}} \\
V^{\frac{1}{2}} L_{c}^{t} & -I
\end{array}\right]<0 .
$$

(iii)

$$
\begin{aligned}
& \left(C_{p}\right)_{i}(Y+Z)\left(C_{p}\right)_{i}^{t} \leq \phi^{2}, i=n_{s}+1, \cdots, n_{s}+m, \\
& \left(C_{p}\right)_{j}(Y+Z)\left(C_{p}\right)_{j}^{t} \leq d \phi^{2}, j=n_{s}+m+1, \cdots, n_{s}+2 m, \\
& \left(C_{p}\right)_{k}(Y+Z)\left(C_{p}\right)_{k}^{t} \leq \alpha^{2}, k=n_{s}+2 m+1, \cdots, n_{s}+3 m-1, \\
& \left(C_{p}\right)_{l}(Y+Z)\left(C_{p}\right)_{l}^{t} \leq d \alpha^{2}, l=n_{s}+3 m, \cdots, n_{s}+4 m-1 .
\end{aligned}
$$

Furthermore, if there exists a feasible solution to LMIs, then the full state feedback gain $K_{c}$ can be given by $K_{c}=G Z^{-1}$.

Proof. The proof utilizes Lemma 1 and the separation principal for state feedback and observer design. Recall the open-loop system $\Sigma_{o}$ described in (21) and the observer-based output feedback controller (22). As in the usual observer design process, we let $e(t)=x_{p}(t)-x_{c}(t)$ to denote error dynamics, then $\dot{e}(t)=$ $\dot{x}_{p}(t)-\dot{x}_{c}(t)$. Substituting (21) and (22) into $\dot{e}(t)$, and replacing $x(t)$ in terms of $e(t)$ and $x_{c}(t)$ in (22), we obtain the feedback-controlled closed-loop system as follows,

$$
\begin{aligned}
& {\left[\begin{array}{c}
\dot{e}(t) \\
\dot{x}_{c}(t)
\end{array}\right]=\underbrace{\left[\begin{array}{cc}
A_{p}-L_{c} M_{p} & 0 \\
L_{c} M_{p} & A_{p}+B_{p} K_{c}
\end{array}\right]}_{A}\left[\begin{array}{c}
e(t) \\
x_{c}(t)
\end{array}\right]+\underbrace{\left[\begin{array}{cc}
D_{p} & -L_{c} \\
0 & L_{c}
\end{array}\right]}_{D} \underbrace{\left[\begin{array}{c}
w(t) \\
v(t)
\end{array}\right]}_{w_{p}}} \\
& y_{i}=\underbrace{\left[\begin{array}{ll}
\left(C_{p}\right)_{i} & \left(C_{p}\right)_{i}
\end{array}\right]}_{C_{i}}\left[\begin{array}{c}
e(t) \\
x_{c}(t)
\end{array}\right]
\end{aligned}
$$

Note that (28) is in the form of (9) and the intensity of $w_{p}(t)$ is given by

$$
W_{p}=\operatorname{diag}\{W, V\}>0 .
$$

Let $X>0$ be defined by

$$
X=\left[\begin{array}{ll}
Y & 0 \\
0 & Z
\end{array}\right],
$$

where $Y>0$ and $Z>0$ solves (24) and (26), respectively. Then, we show that (30) solves (15), hence from Lemma 1 the closed-loop system is stable. To prove this, we replace $(\bar{X}, A, D, W)$ in (15) by $X$ given in (30), $(A, D)$ given in $(28)$, and $W_{p}$ given in (29), to obtain

$$
\begin{aligned}
& \Phi= {\left[\begin{array}{cc}
\left(A_{p}-L_{c} M_{p}\right) Y+Y\left(A_{p}-L_{c} M_{p}\right)^{t} & Y M_{p}^{t} L_{c}^{t} \\
L_{c} M_{p} Y & \left(A_{p}+B_{p} K_{c}\right) Z+Z\left(A_{p}+B_{p} K_{c}\right)^{t}
\end{array}\right] } \\
&+\left[\begin{array}{cc}
D_{p} W D_{p}^{t} & -L_{c} V L_{c}^{t} \\
-L_{c} V L_{c}^{t} & L_{c} V L_{c}^{t}
\end{array}\right] \\
& \Rightarrow \Phi=\left[\begin{array}{c}
\left(A_{p}-L_{c} M_{p}\right) Y+Y\left(A_{p}-L_{c} M_{p}\right)^{t} \\
+D_{p} W D_{p}^{t}+L_{c} V L_{c}^{t} \\
0
\end{array}\right\} 0 \\
&\left.\left\{\begin{array}{c}
\left(A_{p}+B_{p} K_{c}\right) Z+Z\left(A_{p}+B_{p} K_{c}\right)^{t} \\
+L_{c} V L_{c}^{t}
\end{array}\right\}\right] .
\end{aligned}
$$

10 of 18 
The last equality is derived from applying the observer gain $L_{c}$ given in $(25)$. Therefore, $(1,1)$-block matrix of $\Phi$ can be rewritten as

$$
\Phi(1,1)=A_{p} Y+Y A_{p}^{t}-Y M_{p}^{t} V^{-1} M_{p} Y+D_{p} W D_{p}^{t},
$$

and it is the Schur complement of $(24)$, hence $\Phi(1,1)<0$. Let $G=K_{c} Z$, then $\Phi(2,2)$ can be rewritten as

$$
\Phi(2,2)=A_{p} Z+Z A_{p}^{t}+B_{p} G+G^{t} B_{p}^{t}+L_{c} V L_{c}^{t},
$$

and this is the Schur complement of $(26)$, therefore $\Phi(2,2)<0$. Hence, the closed-loop system is stable. To show (27), we recall that the output covariance for closed-loop system (28) is given by

$$
C_{i} X C_{i}^{t}=\left(C_{p}\right)_{i}(Y+Z)\left(C_{p}\right)_{i}^{t}, i=1, \cdots, q .
$$

This completes the proof.

\section{Simulation Results}

We consider the application of proposed LMI-based optimal control law to suppress the fluttering motion of GTM aeroelastic wing. The GTM under consideration is configured with 11 VCCTE flaps in each wing, and at cruising speed of Mach 0.88 and altitude of 35,000 ft. At this condition, the aeroelastic wing has already exhibited fluttering behavior, since in this case both first bending and torsional modes have become unstable; see Table 1 . The relative deflection angle and deflection rate between two adjacent flaps are constrained by, respectively, $2 \mathrm{deg}$ and $60 \mathrm{deg} / \mathrm{sec}$. That is, $\left|\theta_{i}-\theta_{i+1}\right| \leq 2 \mathrm{deg}$ and $\left|\dot{\theta}_{i}-\dot{\theta}_{i+1}\right| \leq 60 \mathrm{deg} / \mathrm{sec}$. Individually, each flap deflection is constrained by $\pm 20 \mathrm{deg}$ and flap deflection rate by $\pm 60 \mathrm{deg} / \mathrm{sec}$. In addition, we assume that the flap actuator dynamics and elastomer have the natural frequency of $10 \mathrm{~Hz}$ and damping ratio of 0.8 .

The aeroelastic model considered in this study includes 6 flexible modes; including two unstable modes. For aeroelastic measurement, we take the outputs at 5 equally spaced data points along the wing span; the last point being at the wing tip. We measure both bending and torsional displacements and rates at these 5 locations; however, only bending and torsional displacements are considered as control outputs. In this study, we assume that GTM is subject to a random gust turbulence of $\pm 30 \mathrm{ft} / \mathrm{sec}$ and random measurement noise of $0.001 \mathrm{ft}^{2}\left(\mathrm{rad}^{2}\right)$. The Dryden's longitudinal wind turbulence model is used, and it is incorporated with aeroelastic model. The objective of bounded control law design then is to suppress the fluttering motion of the wing using VCCTEFs subjected to their actuation constraints. The set of LMIs in Theorem 1 is implemented and solved in MATLAB environment by utilizing SeDuMi as optimization solver and YALMIP as LMI parser. Table 1 contains the list of aeroelastic modal frequency and damping considered in the simulation.

Table 1. Aeroelastic modal data for GTM

\begin{tabular}{|c|c|c|}
\hline Mode ID & Frequency $(\mathrm{rad} / \mathrm{sec})$ & Damping \\
\hline \hline 1st bending & 9.2 & -0.0031 \\
\hline 1st torsion & 17.4 & -0.0487 \\
\hline 2nd bending & 9.71 & 0.22 \\
\hline 3rd bending & 16.1 & 0.216 \\
\hline 4th bending & 26.5 & 0.0369 \\
\hline 2nd torsion & 34.2 & 0.0254 \\
\hline
\end{tabular}

The proposed LMI-based optimal output feedback control law is able to asymptotically stabilize the unstable modes and suppress the fluttering behavior. Figure 5 shows the pole locations for open-loop and closed-loop systems. It is clear that the two open-loop unstable modes are stabilized. Figures 6 and 7 show the wing tip deflection and twist of the closed-loop system, and Table 2 contains the closed-loop output covariance data at the measurement points. It should be noted that these covariance are treated as objective function and subjected to minimization in the optimization process. 


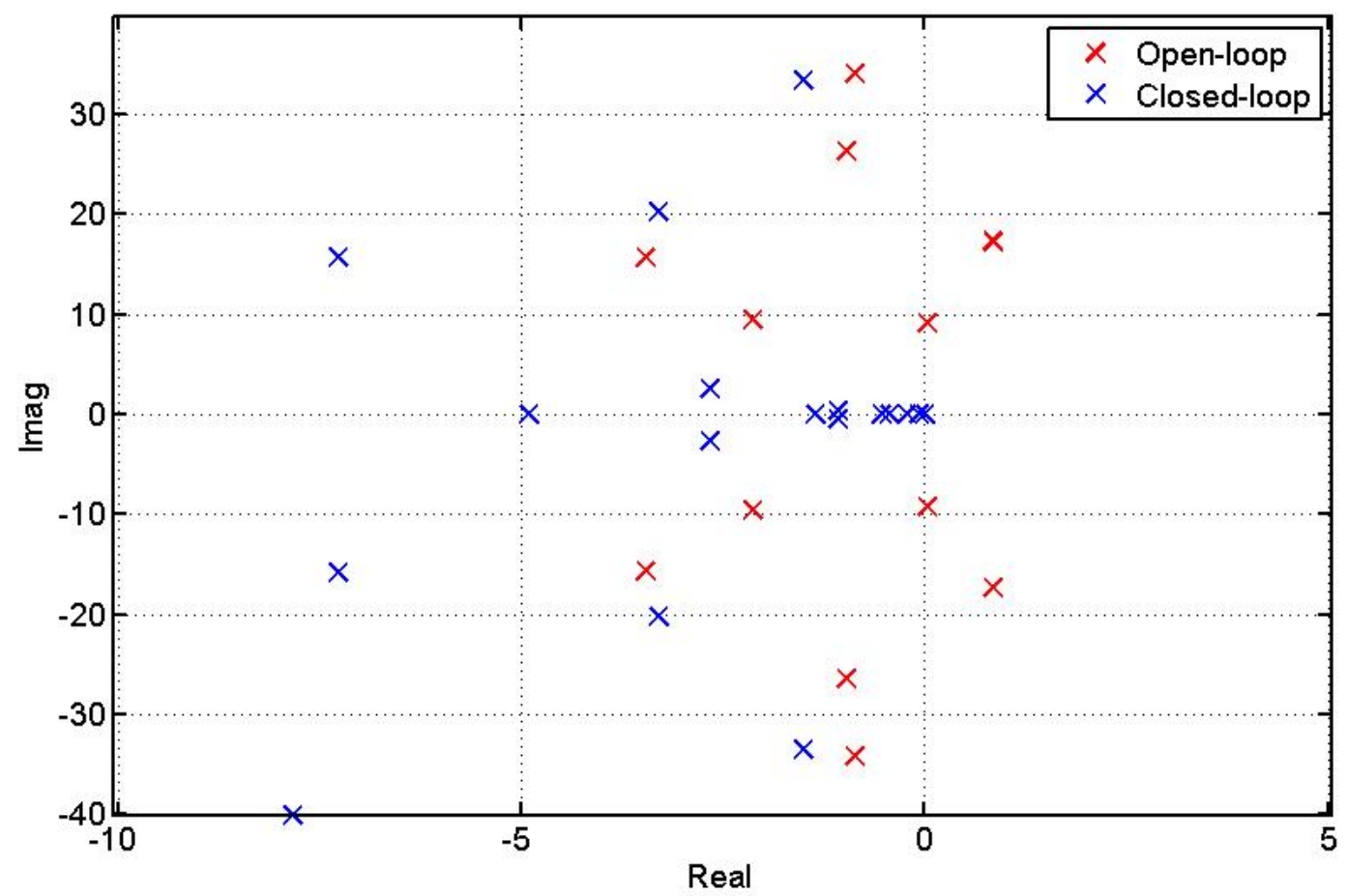

Figure 5. Open-loop and closed-loop pole locations.

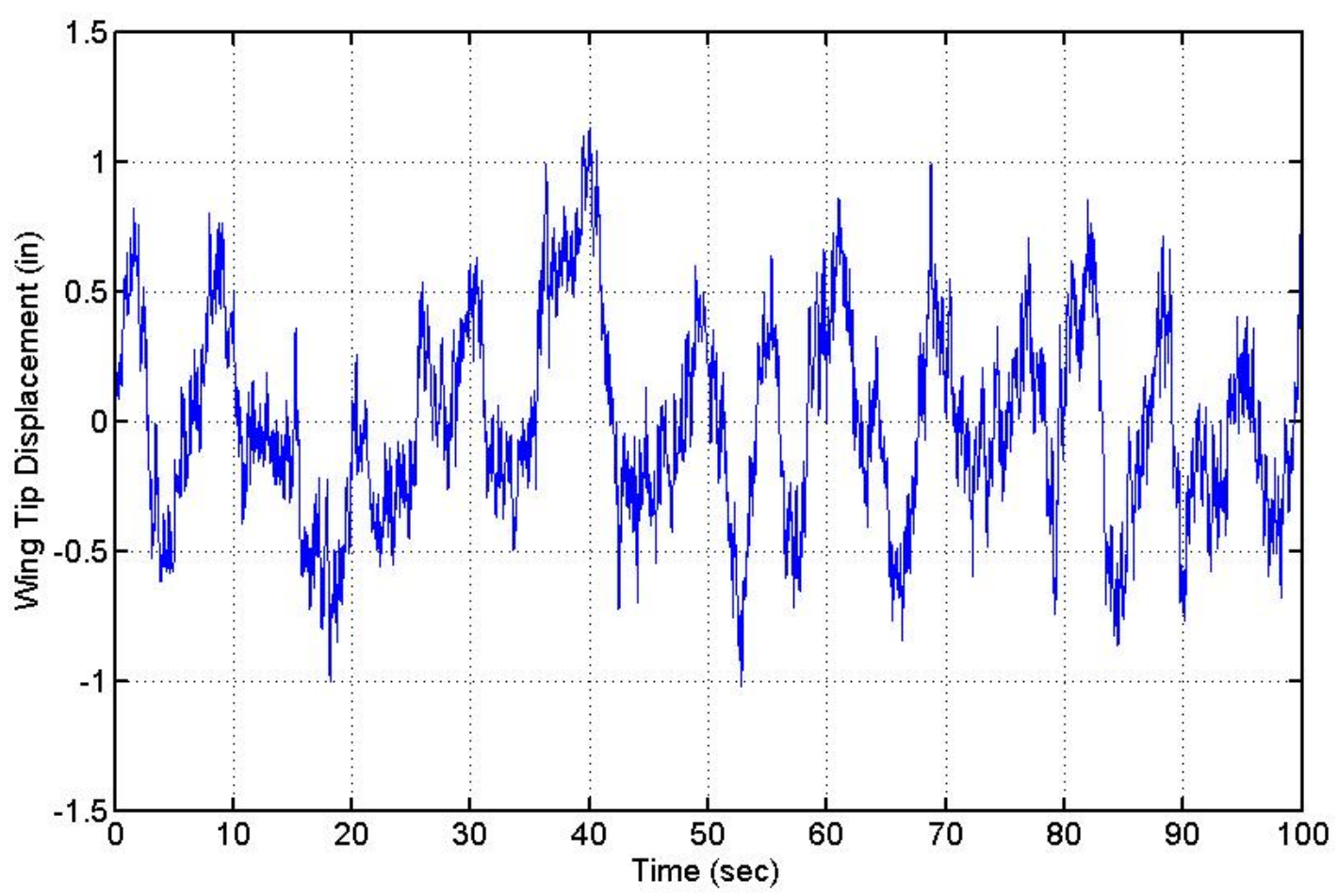

Figure 6. Wing tip deflection response for closed-loop system. 


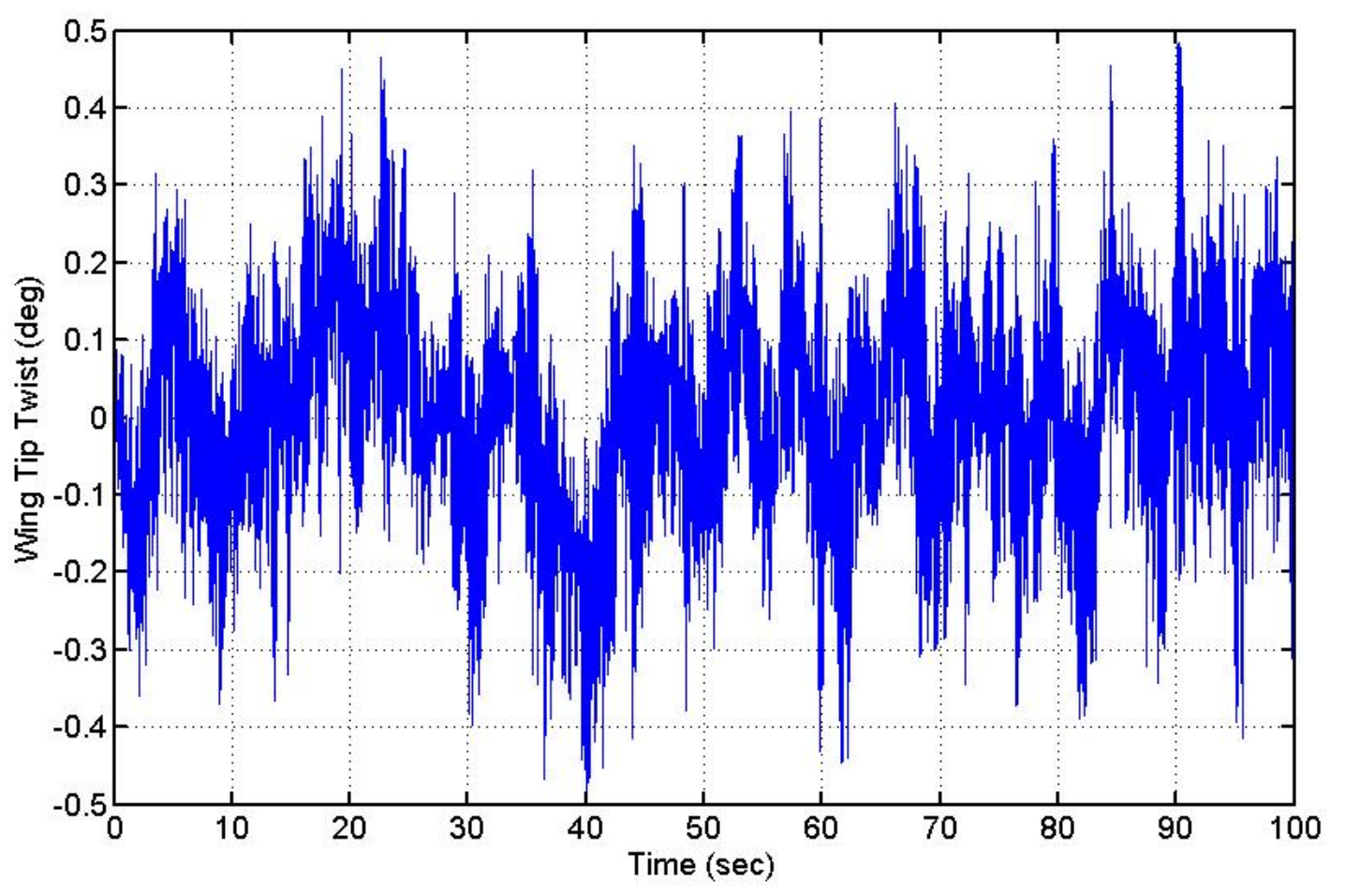

Figure 7. Wing tip twist response for closed-loop system.

Table 2. Closed-loop Output Covariance

\begin{tabular}{|c|c|c|}
\hline Data Point & Bending $\times 10^{-3}\left(\mathrm{ft}^{2}\right)$ & Torsion $\times 10^{-5}\left(\mathrm{rad}^{2}\right)$ \\
\hline \hline Pt. 1 & 0.004 & 0.0008 \\
\hline Pt. 2 & 0.0226 & 0.0451 \\
\hline Pt. 3 & 0.2756 & 0.2007 \\
\hline Pt. 4 & 0.3286 & 0.4497 \\
\hline Pt. 5 & 0.9997 & 0.5997 \\
\hline
\end{tabular}


Figures 8 and 9 display the statistical covariance data showing the relative deflection and deflection rate of the adjacent flaps. Note that the values have been converted to indicate physical quantities and they are all bounded by the given constraints, namely, $2 \mathrm{deg}$ for relative deflection and $60 \mathrm{deg} / \mathrm{sec}$ for relative deflection rate. Furthermore, Figures 10 and 11 show a sample time history data for relative deflection and deflection rate of the two adjacent flaps. Finally, Figures 12 and 13 show the time history of angular deflection and deflection rate for each individual flap.

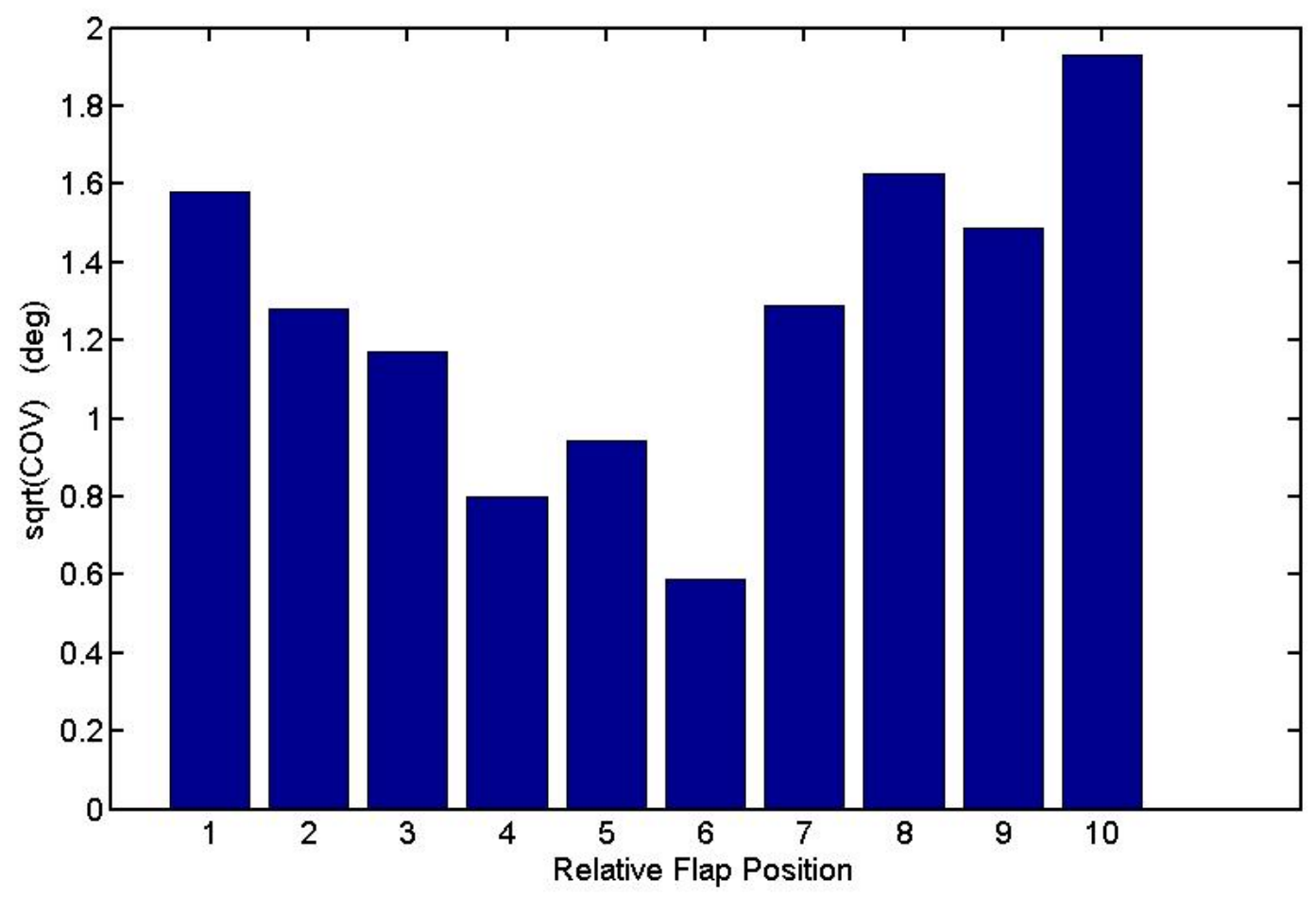

Figure 8. Relative flap deflection.

\section{Conclusions}

In this paper, we presented an LMI-based optimal flight control design concept that utilizes the output covariance control methodology. A series of second order dynamics interconnected with spring and dashpot model was developed to capture the essence of VCCTEF mechanisms and elastomers. Though the stiffness and damping effect for elastomer were assumed to be constant in this study, in actual implementation they can be functions of ambient conditions and can therefore be made parametric dependent. The proposed VCCTEF model was incorporated into the aeroelastic wing model, and the actuation constraints were represented as part of output constraints, hence the standard LMI characterization for the output covariance constraint problem was applied. The conventional observer-based dynamic output feedback control design approach was used, and it was designed separately from the full state feedback design. To demonstrate the efficacy of proposed approach, a GTM model at flutter condition was considered. The simulation results showed that the proposed LMI-based optimal output feedback control law was able to effectively suppress the fluttering motion of the wing, while meeting the VCCTEF actuation constraints.

\section{Acknowledgments}

The authors would like to acknowledge the funding support of NASA ARMD Fixed Wing project and NARI Seedling Fund project. 


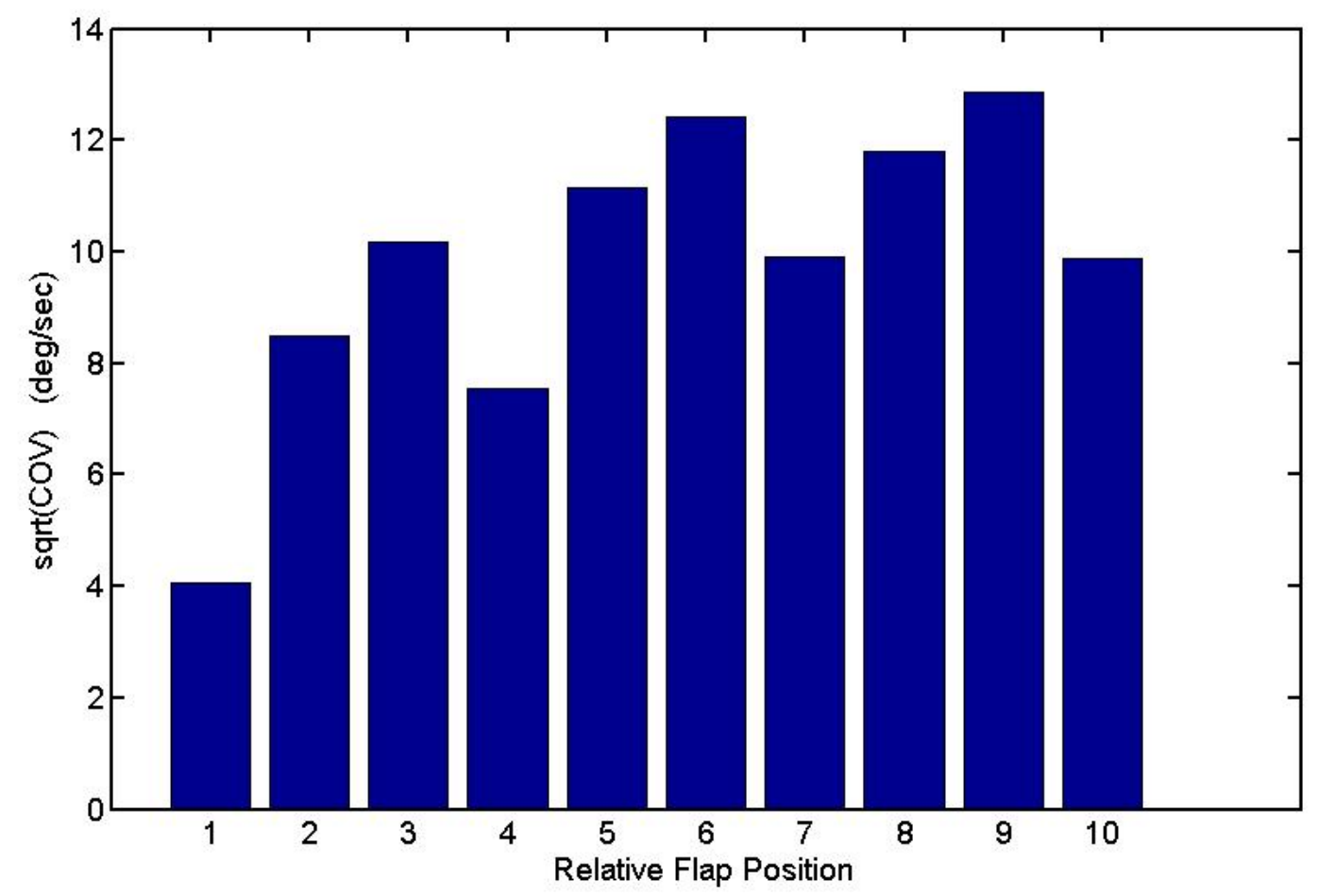

Figure 9. Relative flap deflection rate.

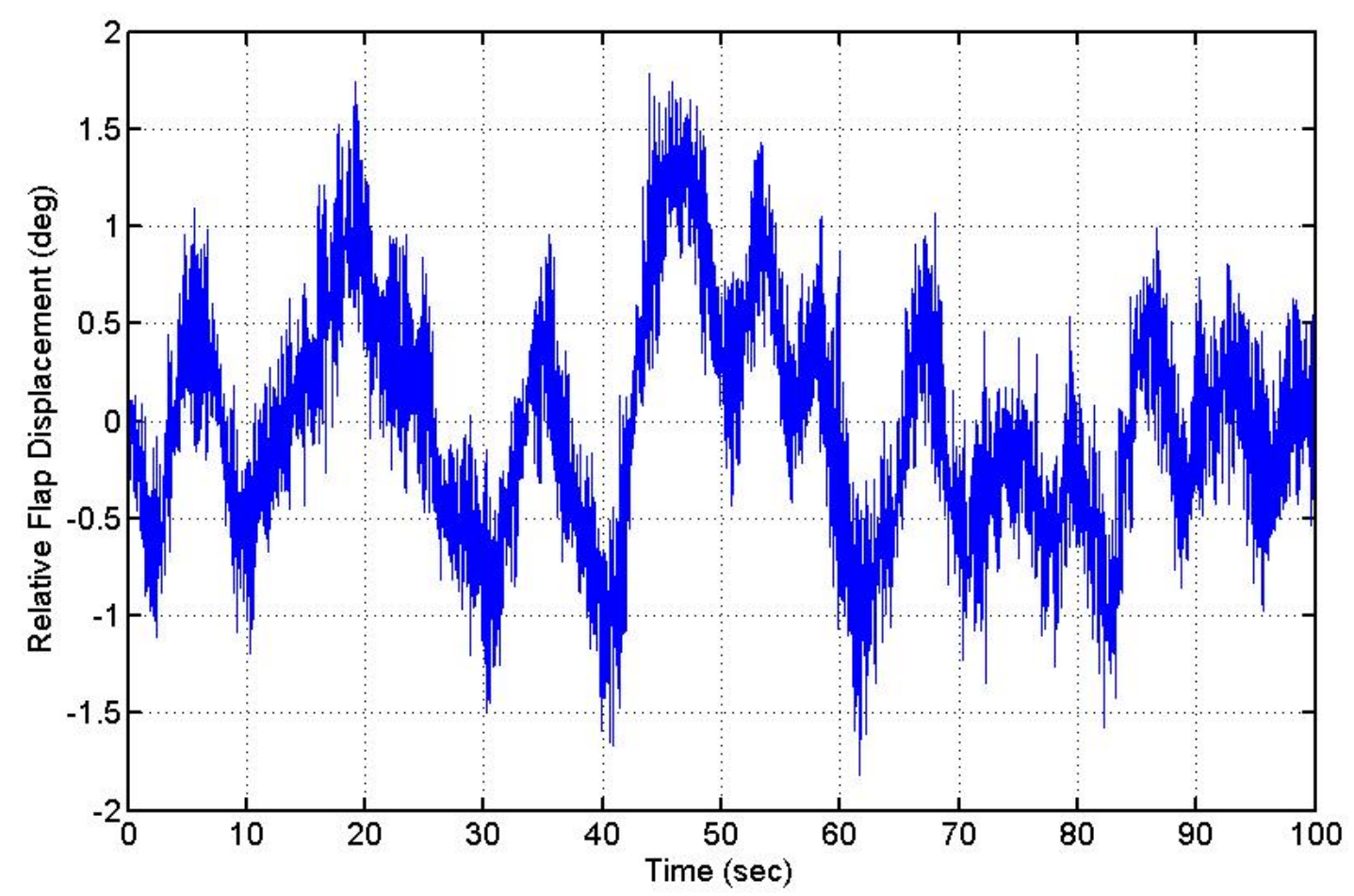

Figure 10. Sample time history for relative flap deflection. 


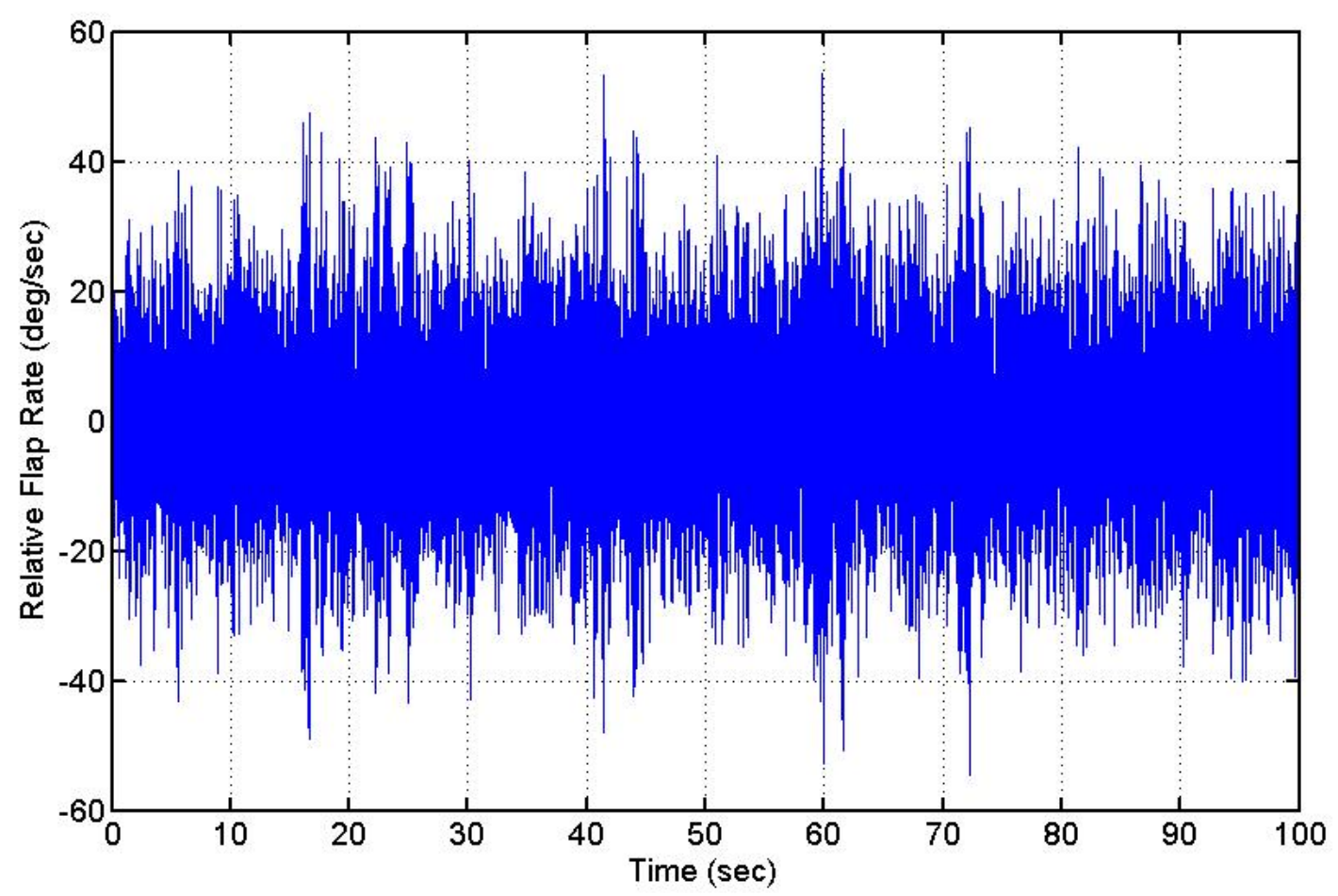

Figure 11. Sample time history for relative flap deflection rate.

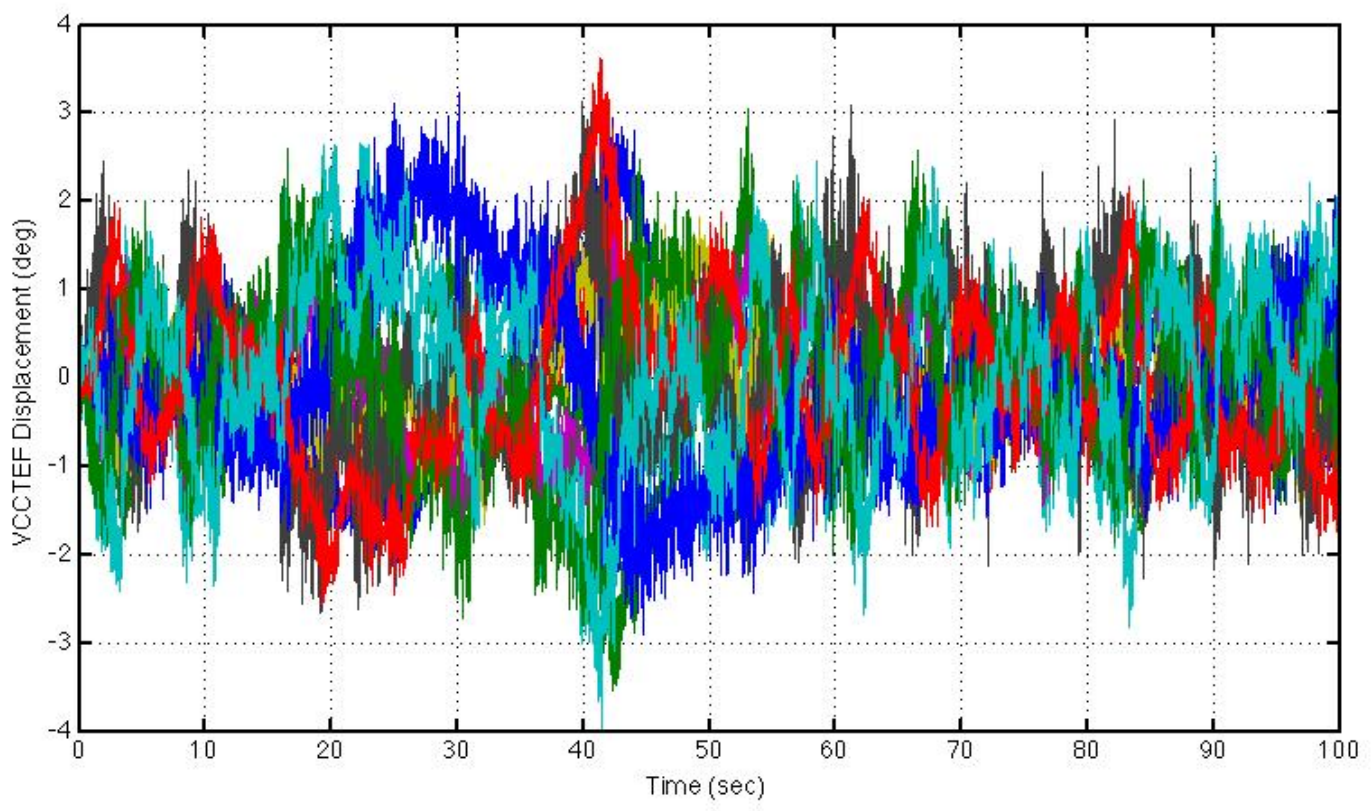

Figure 12. Time history for individual flap deflection. 


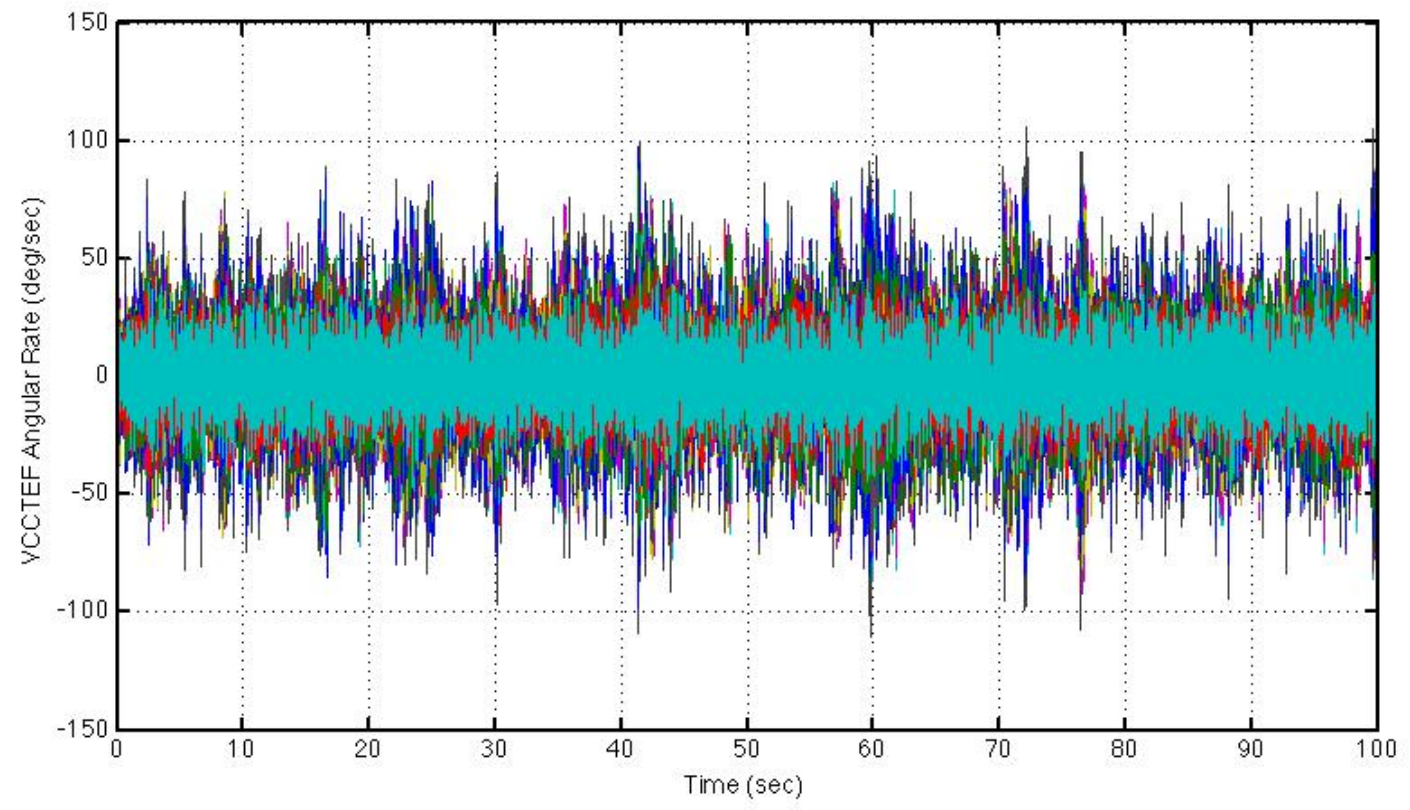

Figure 13. Time history for individual flap deflection rate.

\section{References}

${ }^{1}$ Urnes, J., Nguyen, N., Ippolito, C., Totah, J., Trinh, K., Ting, E., ”'A Mission-Adaptive Variable Camber Flap Control System to Optimize High Lift and Cruise Lif-to-Drag Ratios of Future N+3 Transport Aircraft,"' AIAA Aerospace Sciences Meeting, Grapevine, TX, 2013.

${ }^{2}$ Swei, S., Nguyen, N., "'Aeroelastic Wing Shaping Control Subject to Actuation Constraints,"' 2014 SciTech Conference, National Harbor, MD, 2014

${ }^{3}$ Swei, S., Zhu, G., Nguyen, N., "Integrated Model Reduction and Control of Aircraft with Flexible Wings," AIAA Guidance, Navigation, and Control Conference, Boston, MA, 2013.

${ }^{4}$ Lombaerts, T.J.J., Looye, G.H.N., Chu, Q.P., Mulder, J.A., "'Pseudo Control Hedging and its Application for Safe Flight Envelope Protection,"' AIAA Guidance, Navigation, and Control Conference, Toronto, Ontario, Canada, 2010.

${ }^{5}$ Shewchun, J.M., Feron, E., "High Performance Bounded Control of Systems Subject to Input and Input Rate Constraints,"' AIAA Guidance, Navigation, and Control Conference, New Orleans, LA, 1997.

${ }^{6}$ Hess, R.A., Snell, S.A., "'Flight Control System Design with Rate Saturating Actuators,"' Journal of Guidance, Control, and Dynamics, Vol. 20, No. 1, pp. 90-96, 1997.

${ }^{7}$ Lin, Z., "Semi-global Stabilization of Linear Systems with Position and Rate-limited Actuators,"' Systems E Control Letters, 30, pp. 1-11, 1997.

${ }^{8}$ Johnson, E.N., Calise, A.J., "'Pseudo-Control Hedging: A New Method for Adaptive Control,"' Advances in Navigation Guidance and Control Technology Workshop, 2000.

${ }^{9}$ Nguyen, N., Tuzcu, I., Yucelen, T., and Calise, A., "'Longitudinal Dynamics and Adaptive Control Application for an Aeroelastic Generic Transport Model," AIAA Atmospheric Flight Mechanics Conf., AIAA Paper 2011-6291, Portland, Or., 2011.

${ }^{10} \mathrm{Zhu}$, G., Rotea, M. A., and Skelton, R. E., "'A Convergent Algorithm for the Output Covariance Constraint Control Problem,"' SIAM J. Control Optim., Vol. 35, No. 1, 1997, pp. 341-361.

${ }^{11}$ Kailath, T., Linear Systems, Prentice-Hall, Inc., N.J., 1980.

${ }^{12}$ Nesterov, Y., and Nemirovskii, A., Interior-Point Polynomial Algorithms in Convex Programming, Studies in Applied and Numerical Mathematics, SIAM, Philadelphia, 1994.

${ }^{13}$ Rotea, M. A., "The Generalized $\mathrm{H}_{2}$ Control Problem,"' Automatica, Vol. 29, No. 2, 1993, pp. 373-385.

${ }^{14}$ Skelton, R. E., Dynamics System Control, Wiley, New York, 1988.

${ }^{15}$ Scherer, C., Gahinet, P., and Chilali, M., "'Multiobjective Output-Feedback Control via LMI Optimization,"' IEEE Trans. Auto. Control, Vol. 42, No. 7, 1997, pp. 896-911.

${ }^{16}$ Iwasaki, T., and Skelton, R. E., "All Controllers for the General $H_{\infty}$ Control Problem: LMI Existence Conditions and State Space Formulas,"' Automatica, Vol. 30, No. 8, 1994, pp. 1307-1317.

${ }^{17}$ Gahinet, P., "'A Convex Parametrization of $H_{\infty}$ Suboptimal Controllers,"' 31st Conf. on Decision and Control, Tucson, AZ, 1992, pp. 937-942.

${ }^{18}$ Gahinet, P., and Apkarian, P., "'An LMI-based Parametrization of all $H_{\infty}$ Controllers with Applications,"' 32nd Conf. on Decision and Control, San Antonio, TX, 1993, pp. 656-661. 
${ }^{19}$ White, A., Zhu, G., Choi, J., "'A Linear Matrix Inequality Solution to the Output Covariance Constraint Control Problem,"' ASME Dynamic Systems and Control Conf., Fort Lauderdale, FL, 2012, pp. 163-169. 